\title{
Liraglutide suppresses proliferation and induces adipogenic differentiation of 3T3-L1 cells via the Hippo-YAP signaling pathway
}

\author{
YONGMEI LI ${ }^{1,2^{*}}$, JIANYING DU ${ }^{1 *}$, ENDONG ZHU $^{2}$, JUANJUAN ZHANG ${ }^{2}$, \\ JIE HAN $^{1}$, WEI ZHAO ${ }^{2}$, BEI SUN $^{2}$ and DERUN TIAN ${ }^{1}$ \\ ${ }^{1}$ Department of Human Anatomy and Histology; ${ }^{2}$ Key Laboratory of Hormones and Development (Ministry of Health), \\ Tianjin Key Laboratory of Metabolic Diseases, Tianjin Metabolic Diseases Hospital and Tianjin Institute \\ of Endocrinology, Tianjin Medical University, Tianjin 300070, P.R. China
}

Received July 28, 2017; Accepted December 8, 2017

DOI: $10.3892 / \mathrm{mmr} .2018 .8438$

\begin{abstract}
Liraglutide, as a glucagon-like peptide-1 analogue, is used to treat type 2 diabetes mellitus and obesity. Previous findings have demonstrated the effects of liraglutide on adipogenesis; however, the underlying mechanism involved in this process remains to be elucidated. In the present study, to certify the effect of liraglutide on adipogenesis and explore the possible underlying mechanism involved in this process, preadipocyte 3T3-L1 cells were cultured in adipocyte-inducing medium and treated with liraglutide. Subsequently, the expression levels of the master transcription factors and adipocyte-specific genes were measured by reverse transcription-quantitative polymerase chain reaction and immunoblotting analysis. Lipid droplet production was detected by Oil red O staining. Cell proliferation was determined by a Cell Counting Kit- 8 assay and cell immunofluorescence for $\mathrm{Ki67}$, and apoptosis was assessed by flow cytometry. Next, the expression levels of the core components in the Hippo-yes-associated protein (YAP) signaling pathway as well as YAP-specific target genes were measured. Finally, short interfering RNAs of mammalian ste20 kinase 1/2 (MST1/2), a key protein kinase in the Hippo-YAP pathway, were used to determine whether liraglutide regulated
\end{abstract}

Correspondence to: Professor Derun Tian, Department of Human Anatomy and Histology, Tianjin Medical University, 22 Qi-Xiang-Tai Road, Tianjin 300070, P.R. China

E-mail: tiandr@tmu.edu.cn

Dr Bei Sun, Key Laboratory of Hormones and Development (Ministry of Health), Tianjin Key Laboratory of Metabolic Diseases, Tianjin Metabolic Diseases Hospital and Tianjin Institute of Endocrinology, Tianjin Medical University, 22 Qi-Xiang-Tai Road, Tianjin 300070, P.R. China

E-mail: sun_peipei220@hotmail.com

${ }^{*}$ Contributed equally

Key words: liraglutide, adipocyte, adipogenesis, Hippo-yesassociated protein pathway, 3T3-L1 cells adipogenic differentiation via the Hippo-YAP pathway. It was demonstrated that liraglutide promoted adipogenic differentiation, suppressed proliferation, did not affect apoptosis of 3T3-L1 cells and activated the Hippo-YAP signaling pathway at the initial stage of adipogenesis. Silencing of MST1 counteracted the effect of increasing adipogenesis by liraglutide. These results suggested that liraglutide may activate the Hippo-YAP signaling pathway leading to the inhibition of proliferation of preadipocyte 3T3-L1 cells, and result in cells achieving transformation into mature adipocytes sooner. Taken together, the results of the present study may expand knowledge of the underlying mechanism of liraglutide facilitating adipogenesis, and may contribute to the development of GLP-1 receptor agonists for weight loss and increased insulin sensitivity.

\section{Introduction}

According to the Report on the Status of Nutrition and Chronic Diseases of Chinese residents (2015) (1), the prevalence of diabetes mellitus (DM) reached up to $9.7 \%$ in 2012, which resulted in a heavy social and economic burden on individuals, families and the country (2). Therefore, research regarding the pathogenesis, prevention and therapy of DM is of primary concern. Substantial evidence implicates that obesity is a key risk factor for type $2 \mathrm{DM}$ (T2DM) (3). The root etiology of obesity is chronic energy imbalance, which produces adipocyte hypertrophy and hyperplasia, and these processes lead to adipocyte dysfunction, induce insulin resistance and result in T2DM (4,5). Accordingly, exploring the characteristics of adipocytes may help the treatment of obesity and T2DM.

The Hippo-yes associated protein (YAP) signaling pathway has been identified to serve crucial roles in the regulation of cell proliferation, cell apoptosis and cell differentiation, and may modulate development, organ size, tissue homeostasis and tumorigenesis $(6,7)$. The core of the mammalian Hippo-YAP pathway has been well established. In general, upon activation, the extracellular signals are transduced to the mammalian ste20 kinase $1 / 2$ (MST1/2), then, the complex is assembled by MST1/2 and phosphorylates salvador homolog 1 (Sav1), which activates $\mathrm{S} / \mathrm{T}$ protein kinase 
large tumor suppressor 1/2 (LATS1/2). Activated LATS1/2 in complex with Mob phosphorylates and inhibits two homologous transcription coactivators, namely, YAP and its paralog transcriptional co-activator with PDZ-binding motif (TAZ). Finally, YAP/TAZ regulates the expression of a large number of genes important for cell proliferation (8). Ankyrin repeat domain 1 (ANKRD1), connective tissue growth factor (CTGF) and cysteine rich angiogenic inducer 61 (Cyr61) are well-characterized YAP target genes that regulate cell proliferation (9). Several studies have indicated that the Hippo-YAP signaling pathway is involved in adipogenesis (10-12). At the early phase of adipogenic differentiation, adipogenesis is governed by the activity of a series of key transcription factors, especially peroxisome proliferator-activated receptor $\gamma(\operatorname{PPAR} \gamma)$ and CCAAT/enhancer binding protein $\alpha(\mathrm{C} / \mathrm{EBP} \alpha)$, which coordinates the expression of adipogenic genes characteristic of terminally differentiated adipocytes, including adipocyte protein 2 (aP2) (13).

Liraglutide is a glucagon-like peptide-1 (GLP-1) analogue widely used for the therapy of T2DM, and it is also recommended for the treatment of obesity (14). As an insulinotropic hormone, GLP-1 is secreted by intestinal L cells in response to nutrient ingestion, and acts on the GLP-1 receptor to facilitate glucose-dependent insulin secretion, decreases glucagon levels and improves $\beta$-cell neogenesis (14). GLP-1 and liraglutide have previously been reported to regulate adipocyte formation via extracellular signal-regulated kinase, protein kinase $\mathrm{C}$, protein kinase B and Wnt signaling pathways (15-17). However, it is not clear whether other signaling pathways are involved in the regulation of adipogenic differentiation by liraglutide.

The present study investigated the effects and possible underlying mechanisms of liraglutide on preadipocyte 3T3-L1 cell adipogenesis. These findings may contribute to the development of novel drugs to aid weight loss and increase insulin sensitivity.

\section{Materials and methods}

Reagents. Liraglutide was purchased from Novo Nordisk (West Sussex, UK). Anti- $\beta$-actin (cat. no. A1978-100UL) for the western blotting was purchased from Sigma-Aldrich; Merck KGaA (Darmstadt, Germany). Anti-PPAR $\gamma$ (cat. no. 2435), anti-C/EBP $\alpha$ (cat. no. 2295), anti-aP2 (cat. no. 3544), anti-YAP (cat. no. 14074), anti-phosphorylated (p)-YAP (cat. no. 46931), anti-LATS1 (cat. no. 9153) and anti-MST1 (cat. no. 3682) for western blotting were all purchased from Cell Signaling Technology, Inc. (Danvers, MA, USA). Anti-Ki67 (cat. no. 27309-1-AP) for the immunofluorescence assay was purchased from ProteinTech Group, Inc. (Chicago, IL, USA).

Cell lines and cell culture. Preadipocyte 3T3-L1 cells were cultured in Dulbecco's modified Eagle's medium (HyClone; GE Healthcare Life Sciences, Logan, UT, USA) supplemented with $10 \%$ fetal bovine serum (FBS), $200 \mathrm{U} / \mathrm{ml}$ penicillin and $200 \mathrm{U} / \mathrm{ml}$ streptomycin, and were maintained in a humidified incubator containing $5 \% \mathrm{CO}_{2}$ at $37^{\circ} \mathrm{C}$.

Cell differentiation and treatment. For adipocyte differentiation, 3T3-L1 cells that reached $\sim 90 \%$ confluence were cultured in an adipocyte-inducing medium (AIM; $\alpha$-MEM containing
$10 \%$ FBS, $0.5 \mu \mathrm{M}$ dexamethasone, $0.25 \mathrm{mM}$ methylisobutylxanthine, $5 \mu \mathrm{g} / \mathrm{ml}$ of insulin and $50 \mu \mathrm{M}$ indomethacin) in a humidified atmosphere at $37^{\circ} \mathrm{C}$ with $5 \% \mathrm{CO}_{2}$ for 3 days. For liraglutide treatment, cells were cultured in AIM with $0 \mathrm{nM}$ (vehicle), 10,100 , or 1,000 nM liraglutide.

Reverse transcription-quantitative polymerase chain reaction $(R T-q P C R)$. Total RNA was extracted from cells using TRIzol ${ }^{\circledR}$ reagent (Invitrogen; Thermo Fisher Scientific, Inc., Waltham, MA, USA). A total of $0.5 \mu \mathrm{g}$ RNA of each sample was reverse-transcribed into cDNA with a high-capacity cDNA reverse transcription kit (Thermo Fisher Scientific, Inc.) with the following thermocycling parameters: $25^{\circ} \mathrm{C}$ for $10 \mathrm{~min}, 37^{\circ} \mathrm{C}$ for $2 \mathrm{~h}$ and $85^{\circ} \mathrm{C}$ for $5 \mathrm{~min}$. qPCR was performed using SGExcel Fast SYBR Mixture kits (Sangon Biotech Co., Ltd., Shanghai, China) on the Light Cycler ${ }^{\circledR} 96$ Real-Time PCR system (Roche Diagnostics GmbH, Mannheim, Germany), and the thermocycling parameters were followed as previously described (18), which consisted of 40 cycles $\left(95^{\circ} \mathrm{C}\right.$ for $10 \mathrm{sec}, 60^{\circ} \mathrm{C}$ for $10 \mathrm{sec}$ and $72^{\circ} \mathrm{C}$ for $\left.10 \mathrm{sec}\right)$ following an initial denaturation step $\left(95^{\circ} \mathrm{C}\right.$ for $2 \mathrm{~min}$ ). The mRNA expression level of non-POU-domaincontaining, octamer binding protein (NONO) was used as an internal control as previously reported (19). Relative expression was calculated using the comparative threshold cycle method $\left(2^{-\Delta \Delta \mathrm{Cq}}\right)(20)$. The primer sequences used in the study were as follows: C/EBP $\alpha$ forward, 5'-CTGATTCTTGCCAAACTG AG-3' and reverse, 5'-GAGGAAGCTAAGACCCACTAC-3'; PPAR $\gamma$ forward, 5'-CTTG ACAGGAAAGACAACGG-3' and reverse, 5'-GCTTCTACGGATCGAAACTG-3'; aP2 forward, 5'-AAATCACCGCAGACGACAGG-3' and reverse, 5'-GGC TCATGCCCTTTCATAAAC-3'; ANKRD1 forward, 5'-CTT GATGACCTTCGGTGCG-3' and reverse, 5'-GCTCTTCTG TTGGGAAATGCT-3'; CTGF forward, 5'-TCTCCACCCGAG TTACCAATG-3' and reverse, 5'-TGCAGCCAGAAAGCT CAAAC-3'; Cry61 forward, 5'-GGAAAAGGCAGCTCACTG AAG-3' and reverse, 5'-GCACTCTGGGTTGTCATTGGT A-3'; MST1 forward, 5'-TCCCTCAGGATGGAGACTATG A-3' and reverse, 5'-AAGGCTGGGCTGGTGTTG-3'; NONO forward, 5'-TGCTCCTGTGCCACCTGGT-3' and reverse, 5'-CCGGAGCTGGACGGTTGAAT-3'.

Western blotting. The cells were incubated in AIM or treated with different concentrations of liraglutide $(0,10,100$ or $1,000 \mathrm{nM})$ for 3 days. Following this, treated cells were lysed with radioimmunoprecipitation assay lysis buffer (Beyotime Institute of Biotechnology, Haimen, China) and protein concentration was determined with bicinchoninic acid protein assay. Immunoblotting was performed as previously described (21). Briefly, protein (20 $\mu \mathrm{g} / \mathrm{lane})$ was separated on a $12 \%$ SDS denatured polyacrylamide gel and then transferred onto a polyvinylidene difluoride membrane. The membranes were blocked with $5 \%$ skim milk at room temperature for $1 \mathrm{~h}$, and were subsequently incubated with anti- $\beta$-actin, anti-PPAR $\gamma$, anti-C/EBP $\alpha$, anti-aP2, anti-YAP, anti-p-YAP, anti-LATS1 and anti-MST at $4^{\circ} \mathrm{C}$ overnight (all at 1:1,000). Membranes were washed in PBS with $0.1 \%$ Tween-20 and incubated with horseradish peroxidase (HRP)-conjugated goat anti-mouse IgG and goat anti-rabbit IgG secondary antibodies (1:3,000, cat. no. ZDR-5307 and ZDR-5306; OriGene Technologies, Inc., Beijing, China) according to the manufacturer's instructions. 
Finally, the protein of interest was visualized using Immobilon Western Chemiluminescent HRP substrate (EMD Millipore, Billerica, MA, USA). Intensity analysis of images was performed using ImageJ software (version $1.48 \mathrm{v}$; National Institutes of Health, Bethesda, MD, USA).

Oil red $O$ staining. 3T3-L1 cells that reached $\sim 90 \%$ confluence were cultured in AIM or treated with different concentrations of liraglutide $(0,10,100$ or 1,000 nM) for 3 days. Following this, the treated adipocyte-induced 3T3-L1 cells were gently washed twice with cold PBS, and then fixed in $4 \%$ paraformaldehyde for $10 \mathrm{~min}$ at room temperature. Following this, the cells were washed twice with deionized water, and stained with $60 \%$ saturated Oil Red O for $5 \mathrm{~min}$ at room temperature. For Oil Red O quantification, 4\% IGEPAL CA 630 (Sigma-Aldrich; Merck KGaA) in isopropanol was added to each well, and the plate was then rocked on a shaker for 15 min. Light absorbance by the extracted dye was measured at a wavelength of $520 \mathrm{~nm}$.

Cell immunofluorescence. Adipocyte-induced 3T3-L1 cells were treated with different concentrations of liraglutide $(0,10$, 100 or $1,000 \mathrm{nM}$ ) in a humidified atmosphere at $37^{\circ} \mathrm{C}$ with $5 \% \mathrm{CO}_{2}$ for 2 days using the aforementioned procedure. Cells were fixed in $4 \%$ paraformaldehyde for $15 \mathrm{~min}$ and permeabilized with $1 \%$ Triton X-100 at room temperature for $15 \mathrm{~min}$. Following blocking with $1 \%$ bovine serum albumin at room temperature for $2 \mathrm{~h}$, cells were incubated with rabbit anti-Ki67 (1:200) at $4^{\circ} \mathrm{C}$ for $12 \mathrm{~h}$ and incubated with goat anti-rabbit Alexa Fluor 488 at room temperature for $1 \mathrm{~h}(1: 3,000$, cat. no. A-31565; Invitrogen; Thermo Fisher Scientific, Inc.). The images were acquired using the AV300-ASW confocal microscope (Olympus Corporation, Tokyo, Japan). Image magnification, $\mathrm{x} 100$.

Cell proliferation assay. A Cell Counting Kit (CCK)-8 kit (Beyotime Institute of Biotechnology) was used for the evaluation of cell proliferation. A total of $3 \times 10^{3}$ cells/well were seeded into 96-well plates, which were cultured in AIM medium and treated with different concentrations of liraglutide $(0,0.1,1$, $10,100 \mathrm{nM}$ or $1,10,100 \mu \mathrm{M})$ in a humidified atmosphere at $37^{\circ} \mathrm{C}$ with $5 \% \mathrm{CO}_{2}$ for $48 \mathrm{~h}$. CCK-8 reagent was then added to each well and incubated for $2 \mathrm{~h}$ at $37^{\circ} \mathrm{C}$. The absorbance was measured using a pan-wavelength microplate reader at a wavelength of $450 \mathrm{~nm}$ (SynergyMx; BioTek Instruments, Inc., Winooski, VT, USA).

Cell apoptosis assay by flow cytometry (FCM). Adipocyte-inducing 3T3-L1 cells at $\sim 90 \%$ confluence were treated with different concentrations of liraglutide $(0,10,100$ or $1,000 \mathrm{nM})$ in a humidified atmosphere at $37^{\circ} \mathrm{C}$ with $5 \% \mathrm{CO}_{2}$ for 2 days. Subsequently, the cells were used for apoptosis analysis. For FCM analysis, an Annexin V-APC Apoptosis Detection kit from Tianjin Biotech Co., Ltd. (Beijing, China) was used. According to the manufacturer's instructions, the cells were washed twice with cold PBS and resuspended in $1 \mathrm{X}$ binding buffer at a concentration of $1 \times 10^{6}$ cells $/ \mathrm{ml}$. This solution $(100 \mu \mathrm{l})$ was transferred to a $5 \mathrm{ml}$ culture tube and $5 \mu \mathrm{l}$ FITC-Annexin V and $5 \mu \mathrm{l}$ PI was added. Cells were gently vortexed and incubated for $15 \mathrm{~min}$ at room temperature in the dark. Subsequently, $400 \mu \mathrm{l}$ of $1 \mathrm{X}$ binding buffer was added to each tube and was analyzed by FCM (FACSAria I; BD Biosciences, Franklin Lakes, NJ, USA).

Cell transfection. According to the manufacturer's instructions, transfection was performed using Lipofectamine RNAiMAX in Opti-MEM ${ }^{\circledR}$ I Medium (both from Invitrogen; Thermo Fisher Scientific, Inc.) at $37^{\circ} \mathrm{C}$ for $12 \mathrm{~h}$. Briefly, cells were counted and seeded in 12 well plates at $1.5 \times 10^{5}$ cells/well the day prior to transfection to ensure $\sim 50 \%$ confluency on the day of transfection. For 3T3-L1 cell transfection, $50 \mathrm{nM}$ MST1 siRNA (siMST1) or its negative control (siNC; Shanghai GenePharma Co., Ltd., Shanghai, China) were used. The siRNA sequences were as follows: siMST1-1, 5'-GGGACU AGAAUACCUUCAU-3'; siMST1-2, 5'-GGGAAUAACUGC CAUAGAA-3'; siMST1-3, 5'-GGAGAACUCAGAGGAGGA U-3'; siNC, 5'-UUCUCCGAACGUGUCACGU-3'.

Statistical analysis. The results are expressed as the mean \pm standard deviation. A one-way analysis of variance was used followed by Tukey's or Dunnett's post hoc test to analyze the differences between multiple groups. Statistical analysis was performed using SPSS software, version 17 (SPSS, Inc., Chicago, IL, USA). P<0.05 was considered to indicate a statistically significant difference.

\section{Results}

Liraglutide promotes adipogenic differentiation of 3T3-L1 cells. The authors of the present study previously demonstrated that adipogenic induced 3T3-L1 cells may produce a significant increase in lipid droplet numbers when treated with liraglutide (18). In the current study, the adipocyte formation effect of liraglutide was investigated at the early phase of adipogenic differentiation. Preadipocyte 3T3-L1 cells were cultured in AIM, and were treated with different concentrations of liraglutide $(0,10,100$ or $1,000 \mathrm{nM})$. Following 5 days treatment, Oil Red O staining was performed to examine the lipid droplet production. In a dose-dependent manner, 1,000 nM Liraglutide treatment led to a maximal increase of lipid droplet numbers (34\% increase in Oil Red O staining compared with the vehicle group; $\mathrm{P}<0.05$; Fig. 1A and B). Liraglutide also significantly increased the mRNA expression levels of adipocyte marker genes, including $\mathrm{C} / \mathrm{EBP} \alpha, \operatorname{PPAR} \gamma$ and aP2 compared with the levels in the vehicle group (Fig. 1C-E). When cells were treated with 10, 100 and 1,000 nM liraglutide, the mRNA expression levels of $\mathrm{C} / \mathrm{EBP} \alpha$ increased by 1.23 -fold $(\mathrm{P}<0.05)$, 1.58 -fold $(\mathrm{P}<0.01)$ and 1.63 -fold $(\mathrm{P}<0.01)$, respectively (Fig. 1C), the mRNA expression levels of PPAR $\gamma$ increased by 1.18 -fold $(\mathrm{P}<0.05), 1.54$-fold $(\mathrm{P}<0.001)$ and 1.59-fold $(\mathrm{P}<0.001)$, respectively (Fig. 1D), and the mRNA expression levels of aP2 increased by 1.38 -fold $(\mathrm{P}<0.01), 1.75$-fold $(\mathrm{P}<0.001)$ and 1.96-fold $(\mathrm{P}<0.001)$, respectively (Fig. 1E). Enhanced expression of $\mathrm{C} / \mathrm{EBP} \alpha, \mathrm{PPAR} \gamma$ and $\mathrm{aP} 2$ was further verified by western blot analysis (Fig. 1F). These data suggested that liraglutide accelerates adipogenesis at an early stage of differentiation.

Liraglutide suppresses the proliferation of 3T3-L1 cells and does not affect apoptosis. Considering that there is a stage of mitotic clonal expansion prior to the terminal differentiation 


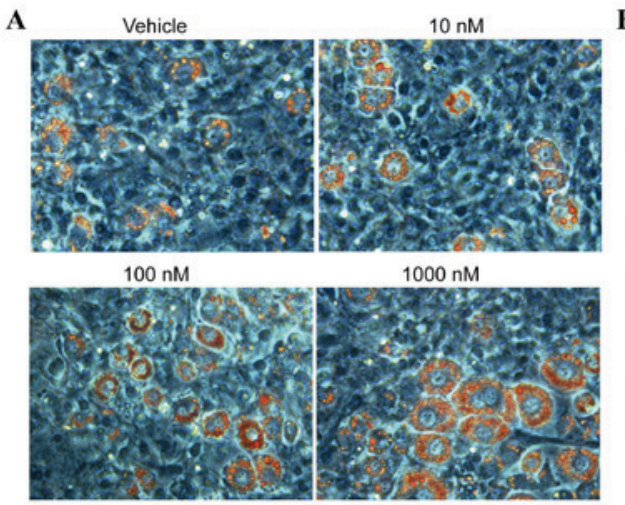

B

C
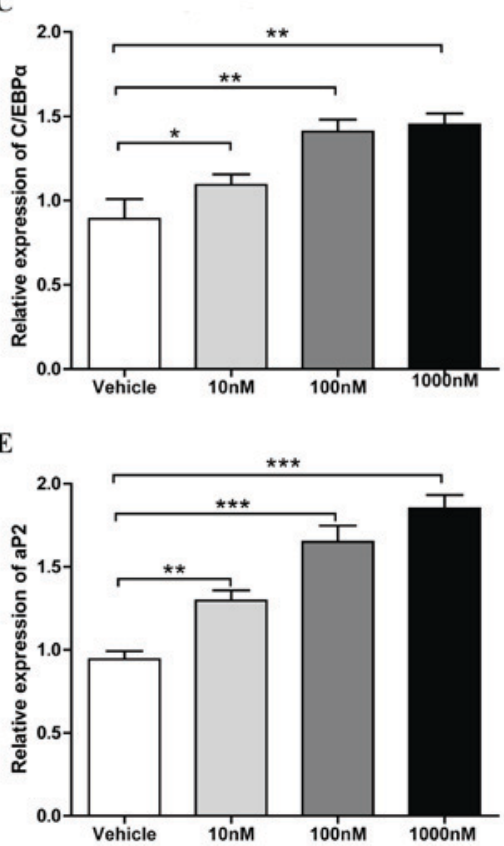

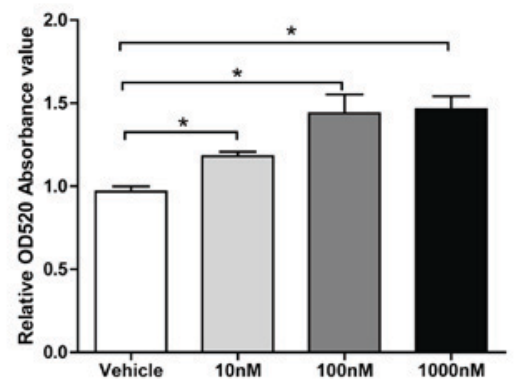

D

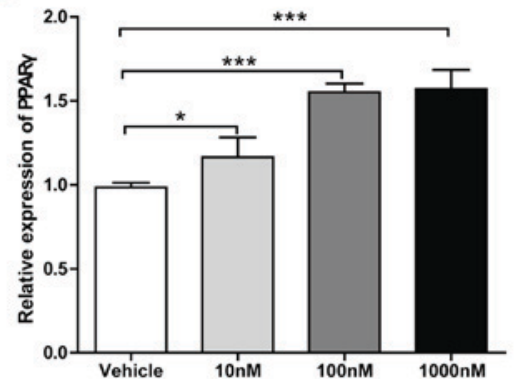

F

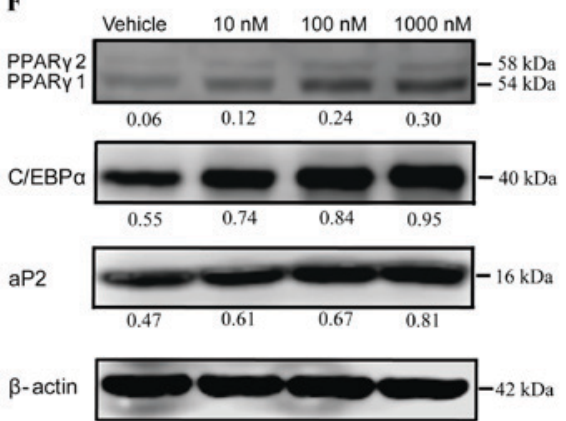

Figure 1. Liraglutide promotes adipogenic differentiation of 3T3-L1 cells. Preadipocyte 3T3-L1 cells were cultured in adipocyte-inducing medium and treated with different concentrations of liraglutide. (A) Representative images of differentiated and treated cells were labeled with Oil Red O at day 5. (B) Oil Red O extracted with isopropanol was measured at OD520, the values represent the mean \pm standard deviation. The mRNA expression levels of key transcription factors, (C) C/EBP $\alpha$ and (D) PPAR $\gamma$, as well as (E) the adipocyte-specific gene aP2, were analyzed by reverse transcription-quantitative polymerase chain reaction at day 2. Results represent the mean \pm standard deviation. (F) The protein expression levels of C/EBP $\alpha$, PPAR $\gamma$ and aP2 were assessed by western blot analysis at day 3 of differentiation. The value under each lane indicates the relative expression level of the genes, which is represented by the intensity ratio between PPAR $\gamma, \mathrm{C} / \mathrm{EBP} \alpha$ or aP2 and $\beta$-actin bands in each lane. $\beta$-actin was used as an internal control. Image magnification, $\mathrm{x} 200$, and all data represent three separate experiments. One-way analysis of variance was used followed by Tukey's post hoc test to analyze the differences between the other groups with the vehicle group in $\mathrm{B}-\mathrm{E} .{ }^{*} \mathrm{P}<0.05,{ }^{* *} \mathrm{P}<0.01$ and ${ }^{* * *} \mathrm{P}<0.001$ vs. vehicle group. OD, optical density; $\mathrm{C} / \mathrm{EBP} \alpha, \mathrm{CCAAT} /$ enhancer binding protein $\alpha$; PPAR $\gamma$, peroxisome proliferator-activated receptor $\gamma$; ap2, adipocyte protein 2 .

stage of adipogenesis, the effect of liraglutide on cell proliferation of 3T3-L1 cells was examined. 3T3-L1 cells were treated with different concentrations of liraglutide $(0,10,100$ or $1,000 \mathrm{nM}$ ) for $48 \mathrm{~h}$, the expression of $\mathrm{Ki} 67$, which is a marker for determining the growth fraction of a given cell population, was determined by immunostaining. As presented in Fig. 2A, the green fluorescence in the cell nucleus treated with different concentrations of liraglutide $(0,10,100$ or $1,000 \mathrm{nM})$ was decreased in a dose-dependent manner. 3T3-L1 cells were treated using the aforementioned procedure and CCK-8 analysis was performed to measure the cell proliferation. The results demonstrated that liraglutide reduced the cell growth in a dose-dependent manner (Fig. 2B). The effect of liraglutide on the cell apoptosis of preadipocyte 3T3-L1 was also examined. 3T3-L1 cells were treated with different concentrations of GLP-1 (0, 10, 100 or 1,000 nM) for $48 \mathrm{~h}$, and cell apoptosis was assessed by FCM assay. As presented in Fig. 2C and D, liraglutide did not affect apoptosis of 3T3-L1 cells compared with the vehicle group. It was also observed that apoptosis of 3T3-L1 cells were not influenced when the cells were induced with AIM and liraglutide following 5 days (data not shown).

Liraglutide activates the Hippo-YAP signaling pathway during adipogenesis. Previous investigations have demonstrated that the Hippo-YAP signaling pathway is involved in cell proliferation and adipogenesis. To clarify whether liraglutide affects this signaling pathway during adipogenesis, the expression of MST1, LATS1, YAP and Ser127 phosphorylated-YAP [p-YAP (S127)] were measured at the early phase of differentiation. 3T3-L1 cells were incubated 
A

DAPI

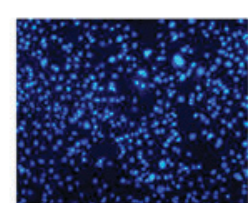

Ki67

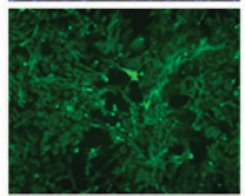

Merge

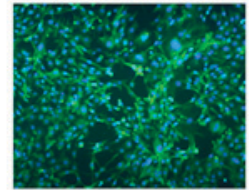

B

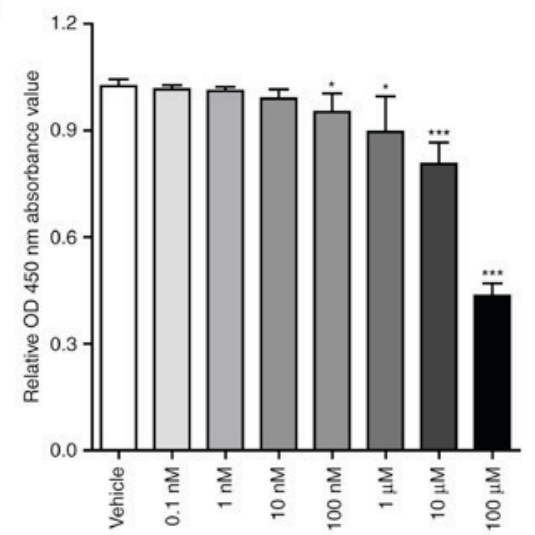

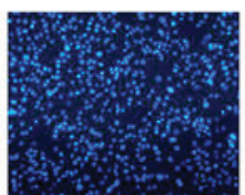
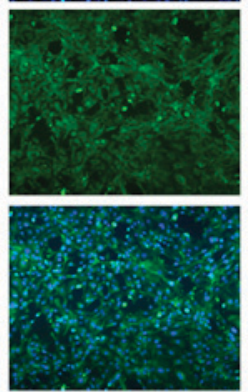

.
C

Liraglutide

$100 \mathrm{nM}$
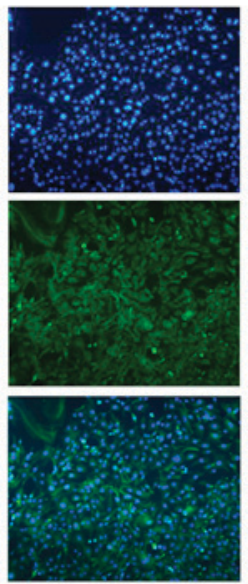
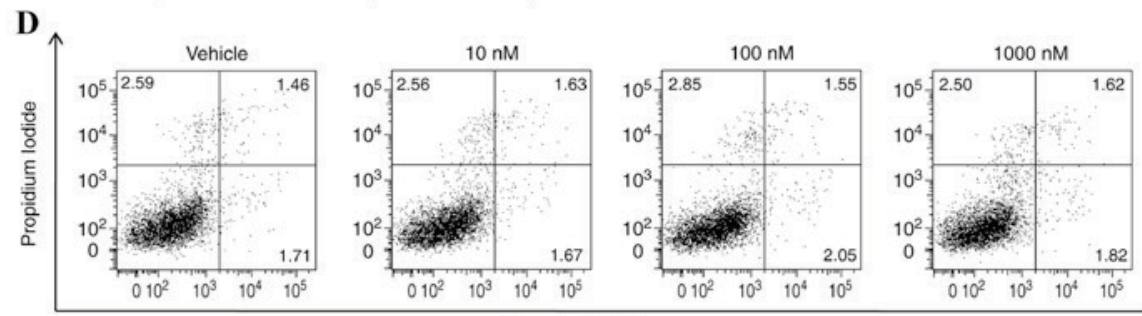

Annexin V-APC

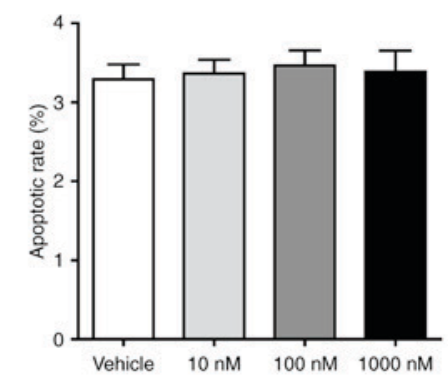

Figure 2. Liraglutide suppresses the proliferation of 3T3-L1 cells and does not affect apoptosis. 3T3-L1 cells were treated with different concentrations of liraglutide for 2 days. (A) Immunostaining for determining cell growth marker gene Ki67 abundance in cell nuclei. Ki67 was labeled as green and nuclei of cells were stained with DAPI as blue. Representative images from three independent experiments are presented, and the image magnification is x100. (B) Cell proliferation was assessed by Cell Counting Kit- 8 assay. The values represent the mean \pm standard deviation. ${ }^{*} \mathrm{P}<0.05$ and ${ }^{* * * *} \mathrm{P}<0.001$ vs. vehicle group. Three separate experiments were performed. (C) The effect of liraglutide on apoptosis was examined by flow cytometric analysis. Cells were stained with Annexin V-APC and propidium iodide. The statistical analysis for the percentage sum of $\mathrm{PI}^{-}$and $\mathrm{PI}^{+}$cells in Annexin $\mathrm{V}^{+}$cells is presented. Data represent the mean \pm standard deviation from three independent experiments. (D) One representative flow cytometric experiment is presented. One-way analysis of variance was used followed by Tukey's post hoc test to analyze the differences between the other groups with the vehicle group in B and C. OD, optical density.

in AIM containing $0,10,100$ and 1,000 $\mathrm{nM}$ liraglutide for $48 \mathrm{~h}$, then the cells were harvested and the expression levels of these proteins was measured by western blotting. As presented in Fig. 3A, no significant alteration in the total protein expression level of YAP was observed, and the levels of MST1, LATS1 and p-YAP (S127) in liraglutide-treated cells were markedly increased compared with vehicle control cells. ANKRD1, CTGF and Cyr61 are well-characterized YAP target genes, and therefore, it was next examined whether liraglutide altered the levels of their expression. Compared with the mRNA expression levels in the vehicle group, when cells were treated with 10, 100 and 1,000 nM liraglutide, the levels of ANKRD1 decreased by 0.56 -fold $(\mathrm{P}<0.01), 0.56$-fold $(\mathrm{P}<0.01)$ and 0.50 -fold $(\mathrm{P}<0.01)$, respectively (Fig. 3B), the levels of CTGF decreased by 0.61 -fold $(\mathrm{P}<0.01), 0.58$-fold $(\mathrm{P}<0.01)$ and 0.73 -fold $(\mathrm{P}<0.05)$, respectively (Fig. 3C), and the mRNA levels of Cyr61 decreased by 0.54-fold $(\mathrm{P}<0.01), 0.60$-fold $(\mathrm{P}<0.01)$ and 0.60 -fold $(\mathrm{P}<0.01)$, respectively (Fig. 3D). These data also suggested that liraglutide may reduce 3T3-L1 cell proliferation.

Silencing of MST1 attenuates liraglutide stimulation of adipogenic differentiation. To further clarify whether the Hippo-YAP signaling pathway mediates liraglutide promotion 
A

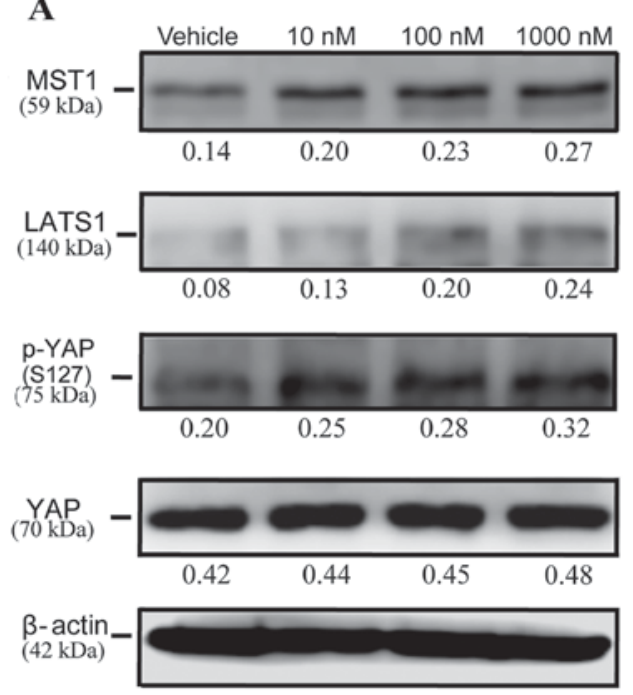

C

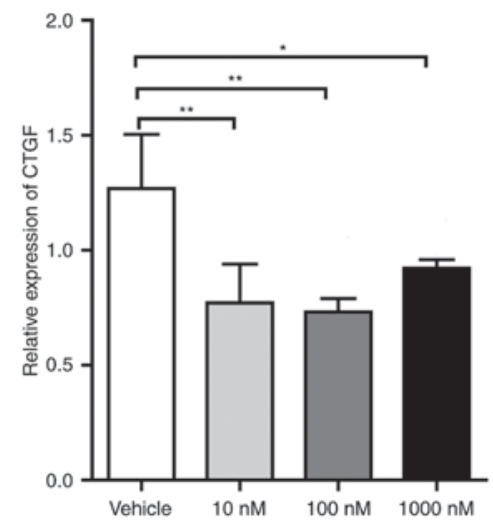

B

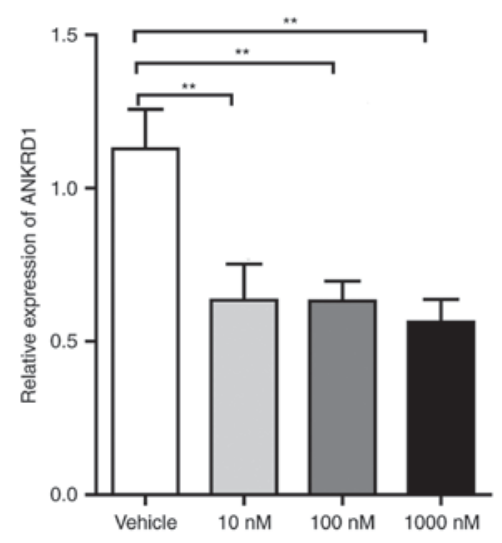

D

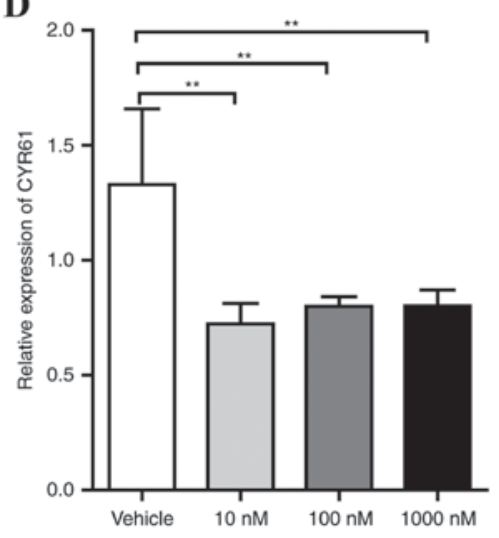

Figure 3. Liraglutide activates the Hippo-YAP signaling pathway during adipogenesis. 3T3-L1 cells were cultured in adipocyte-inducing medium and treated with different concentrations of liraglutide, and then the cells were harvested at day 2. (A) Western blot analysis was used to assess the protein expression levels of the core components of the Hippo-YAP signaling pathway, including MST1, LATS1, p-YAP (S127) and total YAP. The value under each lane indicates the relative expression level of the gene, which is represented by the intensity ratio between MST1, LATS1, p-YAP (S127) or total YAP and $\beta$-actin bands in each lane. $\beta$-actin was used as an internal control. Reverse transcription-quantitative polymerase chain reaction was used to determine the mRNA expression levels of YAP specific target genes, including (B) ANKRD1, (C) CTGF and (D) Cyr61. The values represented the mean \pm standard deviation. All data are representative of at least three independent experiments. One-way analysis of variance was applied followed by Dunnett's test to analyze the differences between the vehicle, 10, 100 and 1,000 $\mathrm{nM}$ groups in B-D. "P<0.05 and ${ }^{* *} \mathrm{P}<0.01$ vs. vehicle group. YAP, Yes-associated protein; p-YAP, phosphorylated yes-associated protein; MST1, mammalian ste20 kinase 1/2; LATS1/2, large tumor suppressor 1/2; ANKRD1, ankyrin repeat domain 1; CTGF, connective tissue growth factor; Cyr61, cysteine rich angiogenic inducer 61.

of adipogenesis, liraglutide gain-of-function studies were performed on the background of MST1 silencing. 3T3-L1 cells were transfected with siMST1s or siNC for $48 \mathrm{~h}$, and as presented in Fig. 4A and B, the mRNA and protein expression levels of MST1 were significantly reduced by all three siMST1s, and the expression of p-YAP (S127) was also decreased by MST1 siRNAs. Subsequently, one of three siMST1s was selected to perform further experiments. 3T3-L1 cells were transfected with siMST1 or siNC for $24 \mathrm{~h}$, and then, the cells were incubated in AIM containing 0 or $100 \mathrm{nM}$ liraglutide. As shown in Fig. 4C and D, knockdown of MST1 partially abrogated liraglutide-enhanced adipogenesis. Consistently, the protein expression levels of adipogenic differentiation marker genes including $\mathrm{C} / \mathrm{EBP} \alpha, \mathrm{PPAR} \gamma$ and aP2 were decreased in the cells transfected with siMST1 and incubated with $100 \mathrm{nM}$ liraglutide, as compared with the cells transfected with siNC and incubated with $100 \mathrm{nM}$ liraglutide (Fig. 4E). These results suggested that the Hippo-YAP signaling pathway may be involved in the process of liraglutide enforced adipogenesis during the early phase of differentiation.

\section{Discussion}

Obesity results in insulin resistance that results in individuals having an increased risk of developing T2DM, and insulin resistance may be partly induced by adipocyte hypertrophy, hyperplasia and dysfunction (5). It has been suggested by clinical studies that treatment with liraglutide controls body weight gain, improves insulin resistance and reduces adipocyte hyperplasia $(22,23)$. A previous study revealed that liraglutide treatment may lead to adipogenic differentiated 3T3-L1 cells to produce a significant increase in lipid droplet numbers at the stage of terminal differentiation (18). The present study focused on the function and underlying mechanism of liraglutide in the regulation of adipogenesis at the early phase. 
A

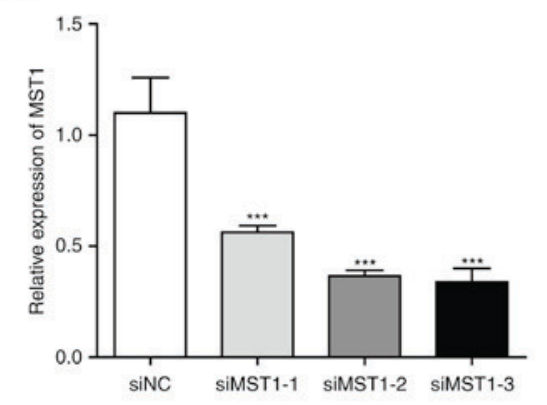

C

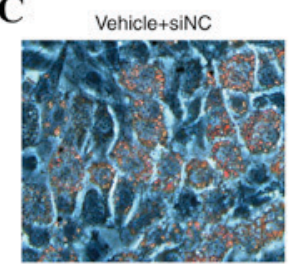

Vehicle+siMST1

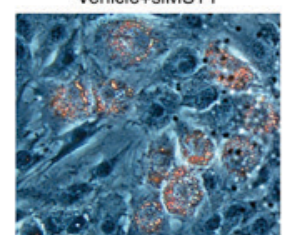

B
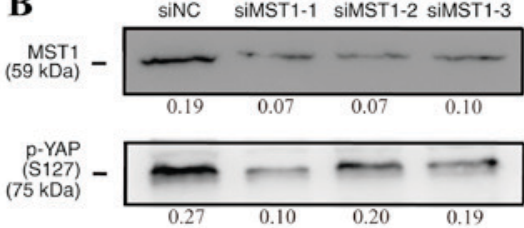

YAP
(70 kDa)

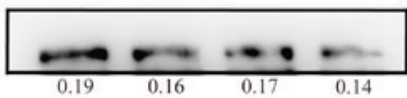

B-actin

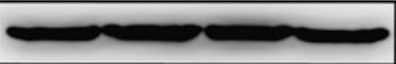

D

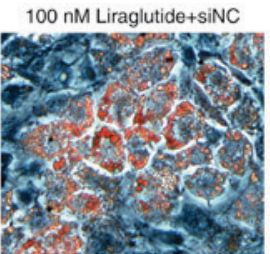

$100 \mathrm{nM}$ Liraglutide+siMST1
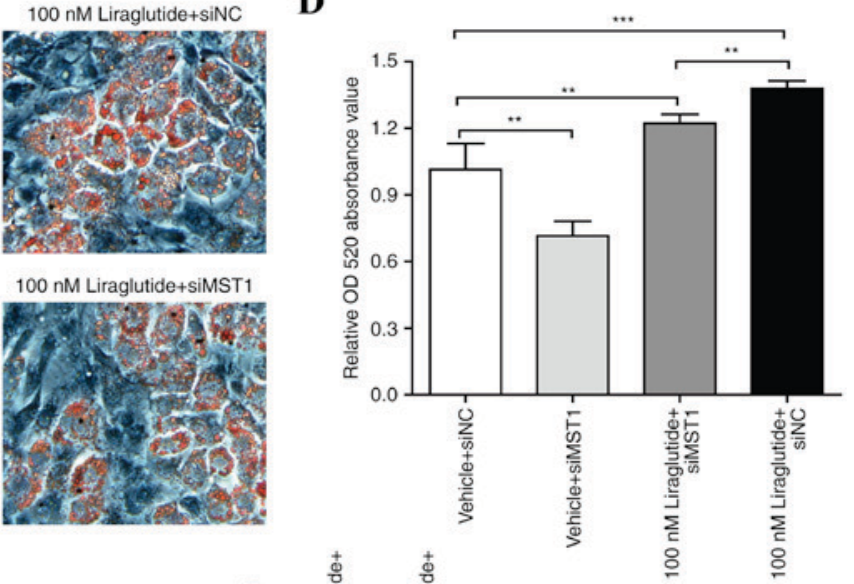
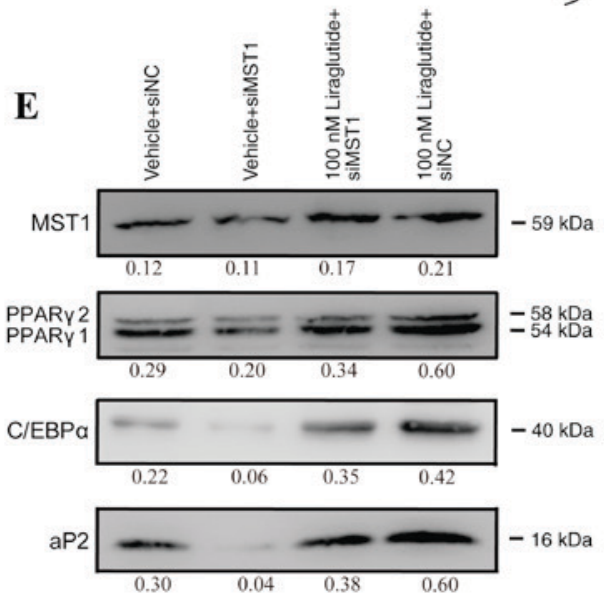

$\beta$-actin

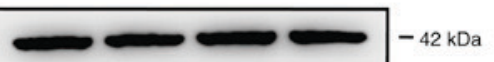

Figure 4. Silencing of MST1 attenuates liraglutide stimulation of adipogenic differentiation. 3T3-L1 cells were transfected with siMST1s or siNC. (A) Following 2 days, reverse transcription-quantitative polymerase chain reaction was used to monitor the mRNA expression levels of MST1, and NONO served as the internal control. The values represent the mean \pm standard deviation. ${ }^{* * *} \mathrm{P}<0.001$ vs. siNC group (B); following 3 days, western blotting was used to measure the protein expression levels of MST1, p-YAP (S127) and total YAP. The value under each lane indicates the relative expression level of the gene, which is represented by the intensity ratio between MST1, p-YAP (S127) or total YAP and $\beta$-actin bands in each lane. $\beta$-actin was used as the internal control. 3T3-L1 cells were transfected with siMST1 or siNC for $24 \mathrm{~h}$, and then, the cells were incubated in adipocyte-inducing medium containing 0 or $100 \mathrm{nM}$ liraglutide. (C) Representative images of differentiated and treated cells were labeled with Oil Red $\mathrm{O}$ at day 5. (D) Oil Red O extracted with isopropanol was measured at OD520, and the values represented the mean \pm standard deviation. ${ }^{* *} \mathrm{P}<0.01$ and ${ }^{* * *} \mathrm{P}<0.001$ vs. vehicle + siNC group. (E) The protein expression levels of MST1, $\mathrm{C} / \mathrm{EBP} \alpha, \mathrm{PPAR} \gamma$ and aP2 were assessed by western blot analysis at day 3 of differentiation. The value under each lane indicates the relative expression level of the gene, which is represented by the intensity ratio between MST1, PPAR $\gamma, \mathrm{C} / \mathrm{EBP} \alpha$ or aP2 and $\beta$-actin bands in each lane. $\beta$-actin was used as an internal control. Image magnification, x200, and all data represented three separate experiments. One-way analysis of variance followed by Tukey's post hoc test was used to analyze the differences between groups with the siNC group in A or the vehicle + siNC group in D. MST1, mammalian ste20 kinase 1; siMST1s, mammalian ste20 kinase 1 short interfering RNA; siNC, negative control short interfering RNA; p-YAP, phosphorylated-yes associated protein; YAP, yes associated protein; OD, optical density; $\mathrm{C} / \mathrm{EBP} \alpha, \mathrm{CCAAT} /$ enhancer binding protein $\alpha$; PPAR $\gamma$, peroxisome proliferator-activated receptor $\gamma$; ap2, adipocyte protein 2.

Adipocytes originate from multipotent mesenchymal stem cells (MSC). During adipogenesis, fibroblast-like pluripotent MSCs differentiate into mature lipid-laden and insulin-responsive adipocytes and the process of adipogenesis involves several stages, which includes mesenchymal precursor and committed preadipocyte proliferation, as well as their differentiation into terminally mature adipocytes (24). This differentiation is tightly regulated by an intricate network 
of transcriptional factors, which is governed to a large extent by an adipocyte-enriched nuclear receptor, PPAR $\gamma$. PPAR $\gamma$ is both necessary and sufficient for adipogenesis, and PPAR $\gamma$ cooperates with $\mathrm{C} / \mathrm{EBPs}$ to induce the expression of numerous downstream target genes important for terminal differentiation including aP2 (13). In the current study, it was observed that liraglutide increased the lipid droplet production of 3T3-L1 cells. It was also demonstrated that liraglutide promoted the expression of the master transcriptional factors $\mathrm{C} / \mathrm{EBP} \alpha$ and PPAR $\gamma$, and enhanced the expression of the downstream adipocyte-specific gene aP2 in a dose-dependent manner. These results suggested that liraglutide may accelerate adipogenesis through a process that upregulates the expression of $\mathrm{C} / \mathrm{EBP} \alpha$ and PPAR $\gamma$ at the early phase of adipogenic differentiation, then promoted the expression of lipogenesis associated genes including aP2, and enhanced the accumulated of lipids.

Next, the present study demonstrated that liraglutide reduced 3T3-L1 cell growth in a dose-dependent manner and did not affect apoptosis at the early phase of adipogenic differentiation. Considering that there is a stage of committed preadipocyte proliferation prior to the terminal differentiation phase of adipogenesis, it was hypothesized that liraglutide may force preadipocyte 3T3-L1 cells to achieve transformation into mature adipocytes earlier, and enhance adipogenesis. Previously, the Hippo-YAP signaling pathway has been demonstrated to have an important role in the regulation of cell proliferation and differentiation. This pathway was initially defined through genetic studies in Drosophila for tumor suppressor genes (6). In mammalian systems, the core components of the Hippo-YAP signaling pathway initiate a kinase cascade, which acts on a transcriptional complex to regulate the expression of target downstream genes that control cell proliferation (25). Briefly, as STE20 family protein kinases, MST1/2 is associated with Sav1/WW45 to phosphorylate Mob1 and LATS1/2. Phosphorylated Mob1 binds to the autoinhibitory motif in LATS1/2, which activates their phosphorylation loop and kinase activity. Next, the active complex (combined LATS1/2 with Mob1) phosphorylates downstream effectors YAP/TAZ, and this leads to their cytoplasmic retention and inhibition. Dephosphorylated YAP/TAZ accumulates in the nucleus and binds to DNA-binding transcription factors to initiate the expression of growth-promoting and apoptosis-inhibiting genes $(7,25)$. Therefore, the active Hippo-YAP signaling pathway induces the cytoplasmic accumulation of phosphorylated YAP and the inhibition of growth promoting genes. In the present study, it was demonstrated that liraglutide increased the levels of the core components of the Hippo-YAP signaling pathway, including MST1, LATS1 and p-YAP (S127) at the early phase of adipogenesis. Consistently, YAP specific target genes were downregulated in liraglutide-treated 3T3-L1 cells, including ANKRD1, CTGF and Cyr61. Silencing of MST1 reduced adipogenic differentiation of 3T3-L1 cells, and silencing of MST1 counteracted the effect of increasing adipogenesis by liraglutide. Previous studies have reported that MST2 interacts with Sav1 to activate PPAR $\gamma$ and augments PPAR $\gamma$-induced adipocyte differentiation (26). LATS2 phosphorylated YAP and TAZ and retained them in the cytoplasm, leading to the reduction of cell proliferation and the promotion of cell adipogenic differentiation (10). The results of the present study and previous studies suggest that the activation of the Hippo-YAP signaling pathway may be involved in the process of liraglutide enhanced adipogenic differentiation.

In conclusion, the present study demonstrated that liraglutide promoted adipogenic differentiation of preadipocyte 3T3-L1 cells. In addition, liraglutide may activate the Hippo-YAP signaling pathway leading to proliferation inhibition of committed preadipocyte, and accordingly, 3T3-L1 cells achieve transformation into mature adipocytes sooner. The results may help to expand the knowledge regarding the underlying mechanism of liraglutide facilitating adipogenesis, and may provide a theoretical support for liraglutide in T2DM and obesity treatment.

\section{Acknowledgements}

The present study was supported by the National Natural Science Foundation of China (grant nos. 81501846 and 81270927), the Scientific Foundation of Tianjin Medical University (grant no. 2014KYM16), the Scientific Foundation of Tianjin Metabolic Diseases Hospital and Tianjin Institute of Endocrinology, Tianjin Medical University (grant no. 2014RC01) and the Tianjin Municipal Natural Science Foundation of China (grant no. 16JCYBJC26800).

\section{References}

1. Yu D, He Y, Guo Q, Fang H, Xu X, Fang Y, Li J and Zhao L: Trends of energy and nutrients intake among Chinese population in 2002-2012. J Hygiene Res 45: 527-533, 2016.

2. Zhang N, Du SM and Ma GS: Current lifestyle factors that increase risk of T2DM in China. Eur J Clin Nutr 71: 832-838, 2017.

3. Flier JS: Obesity wars: Molecular progress confronts an expanding epidemic. Cell 116: 337-350, 2004

4. Guilherme A, Virbasius JV, Puri V and Czech MP: Adipocyte dysfunctions linking obesity to insulin resistance and type 2 diabetes. Nat Rev Mol Cell Biol 9: 367-377, 2008.

5. de Ferranti S and Mozaffarian D: The perfect storm: Obesity, adipocyte dysfunction, and metabolic consequences. Clin Chem 54: 945-955, 2008.

6. Zhao B, Li L, Lei Q and Guan KL: The Hippo-YAP pathway in organ size control and tumorigenesis: An updated version. Genes Dev 24: 862-874, 2010.

7. Yu FX and Guan KL: The Hippo pathway: Regulators and regulations. Genes Dev 27: 355-371, 2013.

8. Zhang Y, Park HW, Jewell JL, Chen Q, Deng Y, Pan D, Taylor SS Lai ZC and Guan KL: Protein kinase A activates the Hippo pathway to modulate cell proliferation and differentiation. Genes Dev 27: 1223-1232, 2013.

9. Zhao B, Panupinthu N, Jewell JL, Lian I, Wang LH, Zhao J, Yuan H, Tumaneng K, Li H, Fu XD, et al: Regulation of the Hippo-YAP pathway by G-protein-coupled receptor signaling. Cell 150: 780-791, 2012

10. An Y, Kang Q, Zhao Y, Hu X and Li N: Lats2 modulates adipocyte proliferation and differentiation via hippo signaling. PLoS One 8: e72042, 2013.

11. Seo E, Basu-Roy U, Gunaratne PH, Coarfa C, Lim DS, Basilico C and Mansukhani A: SOX2 regulates YAP1 to maintain stemness and determine cell fate in the osteo-adipo lineage. Cell Rep 3: 2075-2087, 2013.

12. Chen SN, Gurha P, Lombardi R, Ruggiero A, Willerson JT and Marian AJ: The hippo pathway is activated and is a causal mechanism for adipogenesis in arrhythmogenic cardiomyopathy. Circ Res 114: 454-468, 2014.

13. Ali AT, Hochfeld WE, Myburgh R and Pepper MS: Adipocyte and adipogenesis. Eur J Cell Biol 92: 229-236, 2013.

14. Russell-Jones D: Molecular, pharmacological and clinical aspects of liraglutide, a once-daily human GLP-1 analogue. Mol Cell Endocrinol 297: 137-140, 2009. 
15. Challa TD, Beaton N, Arnold M, Rudofsky G, Langhans W and Wolfrum C: Regulation of adipocyte formation by GLP-1/GLP-1R signaling. J Biol Chem 287: 6421-6430, 2012.

16. Yang J, Ren J, Song J, Liu F, Wu C, Wang X, Gong L, Li W, Xiao F, Yan F, et al: Glucagon-like peptide 1 regulates adipogenesis in 3T3-L1 preadipocytes. Int J Mol Med 31: 1429-1435, 2013.

17. Liu R, Li N, Lin Y, Wang M, Peng Y, Lewi K and Wang Q: Glucagon like peptide-1 promotes adipocyte differentiation via the Wnt 4 mediated sequestering of beta-catenin. PLoS One 11: e0160212, 2016.

18. Zhu E, Yang Y,Zhang J,Li Y,Li C, Chen L and Sun B: Liraglutide suppresses obesity and induces brown fat-like phenotype via Soluble Guanylyl Cyclase mediated pathway in vivo and in vitro. Oncotarget 7: 81077-81089, 2016.

19. Arsenijevic T, Grégoire F, Delforge V, Delporte C and Perret J: Murine 3T3-L1 adipocyte cell differentiation model: Validated reference genes for qPCR gene expression analysis. PLoS One 7: e37517, 2012

20. Livak KJ and Schmittgen TD: Analysis of relative gene expression data using real-time quantitative PCR and the 2(-Delta Delta C(T)) method. Methods 25: 402-408, 2001.

21. Zhu E, Wang X, Zheng B, Wang Q, Hao J, Chen S, Zhao Q, Zhao L, Wu Z and Yin Z: miR-20b suppresses Th17 differentiation and the pathogenesis of experimental autoimmune encephalomyelitis by targeting ROR $\gamma$ t and STAT3. J Immunol 192: 5599-5609, 2014
22. Astrup A, Rössner S, Van Gaal L, Rissanen A, Niskanen L, Al Hakim M, Madsen J, Rasmussen MF and Lean ME; NN8022-1807 Study Group: Effects of liraglutide in the treatment of obesity: A randomised, double-blind, placebo-controlled study. Lancet 374: 1606-1616, 2009.

23. Ng SY and Wilding JP: Liraglutide in the treatment of obesity. Expert Opin Biol Ther 14: 1215-1224, 2014.

24. Lefterova MI and Lazar MA: New developments in adipogenesis. Trends Endocrinol Metab 20: 107-114, 2009.

25. Gumbiner BM and Kim NG: The Hippo-YAP signaling pathway and contact inhibition of growth. J Cell Sci 127: 709-717, 2014.

26. Park BH, Kim DS, Won GW, Jeon HJ, Oh BC, Lee Y, Kim EG and Lee YH: Mammalian ste20-like kinase and SAV1 promote 3T3-L1 adipocyte differentiation by activation of PPAR $\gamma$. PLoS One 7: e30983, 2012. Attribution 4.0 International (CC BY-NC 4.0) License 